\section{Mixed Coordinate Tracking of Generalized Maneuvering Targets Using Acceleration and Jerk Models}

In a recent paper the authors introduced an all-Cartesian formulation of a jerk model for tracking highly maneuvering targets. Here a more complex but realistic case is considered, where target motion modeling and tracking are carried out in the 3-D Cartesian frame using measurements obtained in a spherical system. The transformation of the measurements into the Cartesian system results in nonlinear measurement equations. We solve the problem using an extended Kalman filter (EKF) approach, and also treat the earlier acceleration model similarly for comparison of results.

\section{INTRODUCTION}

In many practical applications it is necessary to accurately track objects with generalized motion, i.e., with trajectories that have significant position derivatives of several orders. A Kalman filter (KF) or its variant is commonly employed for tracking maneuvering targets (see e.g. [1]). For such tracking it is necessary to model the target motion in an appropriate way. One common approach to modeling the kinematics is by using the first few derivatives of the target displacement. The acceleration models (see e.g. [2]) currently used for tracking agile targets use terms only up to the second derivative. However, such models are not adequate to accurately describe the motion of targets with significant position derivatives of order higher than the acceleration.

The problem of model inaccuracy may be alleviated by increasing the order of the kinematic model of the target to include position derivatives up to the jerk or the rate of acceleration. Such an attempt has been made in the case of the simpler $\alpha-\beta-\Gamma$ tracker $[3,4]$. The first attempt at developing a jerk model for use with KF-based tracking has been reported by the present authors in a recent paper [5] which dealt with linear 3-dimensional tracking involving both target model and measurements in Cartesian space.

In this work, the jerk model is applied to a more complex but realistic case of mixed coordinate tracking. Here, the kinematics of the target are

Manuscript received August 2, 1996; revised February 1, 1999 and February 23, 2000; released for publication April 10, 2000.

IEEE Log No. T-AES/36/3/07817.

Refereeing of this contribution was handled by X. R. Li.

$0018-9251 / 00 / \$ 10.00$ (C) 2000 IEEE

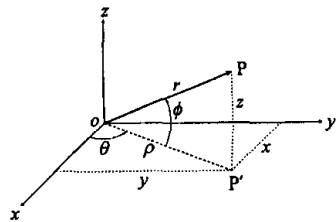

Fig. 1. Cartesian and polar coordinate systems.

modeled in the Cartesian $(x, y, z)$ coordinates, but the target position measurements are assumed to be made in the spherical system, consisting of range $r$, azimuth angle $\theta$, and elevation angle $\phi$, as obtained from a typical monostatic radar system. The coordinate systems are shown in Fig. 1. The transformation of the vector measurement equation from the spherical to the Cartesian coordinate system renders it nonlinear in the Cartesian variables. The nonlinearity is handled here through an extended Kalman filter (EKF) approach in which the equation that expresses the radar measurements in terms of the state vector is expanded about the predicted state by using a Taylor series, and ignoring the second and higher order terms. The resulting approximate measurement equation is linear, but the observation or measurement matrix becomes time varying in nature, and is calculated in every iteration from the predicted state variables.

The degradation due to the approximate linearization process manifests itself in lower tracking accuracies and, in extreme cases, loss of track. In literature there are two methods to circumvent this problem. The first [6] helps prevent divergence by iterating the estimates three or four times in each measurement cycle. The second method [7] improves tracking accuracies by dividing the single measurement process of range, azimuth, and elevation into three sequential subprocesses, with the azimuth first measured and processed through an EKF, followed by elevation and range in succession.

A number of authors (e.g. [8-13]) have dealt with nonlinear tracking problems, but they have generally made use of either kinematic models of second order (acceleration models) or measurements of orientations of the target vehicle. The main contribution of the current work is to consider a higher order kinematic model (jerk model) for target tracking using only position measurements as obtained from normal radars. The jerk model is compared with the acceleration model, which is also formulated and analyzed here under mixed coordinate tracking conditions.

\section{JERK MODEL AND JERK FILTER}

\section{A. System Equations}

A jerk model for target kinematics was introduced by the authors in [5] as an extension of [2]. For 
3-dimensional motion, the discrete model in [5] can be written as

$$
\mathbf{X}_{J}(k+1)=\mathbf{F}_{J}(k) \mathbf{X}_{J}(k)+\mathbf{W}_{J}(k)
$$

where

$$
\begin{aligned}
\mathbf{X}_{J} & =\left[\begin{array}{llllllllllll}
x & \dot{x} & \ddot{x} & \dddot{x} & y & \dot{y} & \ddot{y} & \dddot{y} & z & \dot{z} & \ddot{z} & \dddot{z}
\end{array}\right]^{\mathrm{T}} \\
\mathbf{W}_{J} & =\left[\begin{array}{llllllll}
u_{1 J} & u_{2 J} & u_{3 J} & u_{4 J} & u_{5 J} & u_{6 J} & u_{7 J} \\
& & u_{8 J} & u_{9 J} & u_{10 J} & u_{11 J} & u_{12 J}
\end{array}\right]^{\mathrm{T}} .
\end{aligned}
$$

The terms $u_{1 J}, u_{2 J}$, etc., (1b) are the driving white noise components for the state equation of the jerk model. The state transition matrix for the jerk filter is given by [5]

$$
\mathbf{F}_{J}=\left[\begin{array}{lll}
\mathbf{T} & \mathbf{0} & \mathbf{0} \\
\mathbf{0} & \mathbf{T} & \mathbf{0} \\
\mathbf{0} & \mathbf{0} & \mathbf{T}
\end{array}\right]
$$

where 0 represents a $4 \times 4$ null matrix, and

$$
\mathbf{T}=\left[\begin{array}{cccc}
1 & T & T^{2} / 2 & p_{1} \\
0 & 1 & T & q_{1} \\
0 & 0 & 1 & r_{1} \\
0 & 0 & 0 & s_{1}
\end{array}\right]
$$

In (2a) $T$ is the sample interval between updates, and

$$
\begin{aligned}
& p_{1}=\left(2-2 \alpha T+\alpha^{2} T^{2}-2 e^{-\alpha T}\right) /\left(2 \alpha^{3}\right) \\
& q_{1}=\left(e^{-\alpha T}-1+\alpha T\right) / \alpha^{2} \\
& r_{1}=\left(1-e^{-\alpha T}\right) / \alpha \\
& s_{1}=e^{-\alpha T} .
\end{aligned}
$$

The process noise covariance of the jerk model is

$$
\mathbf{Q}_{J}(k)=2 \alpha \sigma_{J}^{2}\left[\begin{array}{lll}
\mathbf{Q} & \mathbf{0} & \mathbf{0} \\
\mathbf{0} & \mathbf{Q} & \mathbf{0} \\
\mathbf{0} & \mathbf{0} & \mathbf{Q}
\end{array}\right]
$$

where $\sigma_{J}^{2}$ is the variance of the target jerk, $\alpha$ is the Heciprocal of the jerk decorrelation time, and $\mathbf{Q}$ is a $4 \times 4$ symmetric matrix with the following elements:

$$
\begin{aligned}
q_{11}= & \frac{1}{2 \alpha^{7}}\left(\frac{\alpha^{5} T^{5}}{10}-\frac{\alpha^{4} T^{4}}{2}+\frac{4 \alpha^{3} T^{3}}{3}-2 \alpha^{2} T^{2}\right. \\
& \left.+2 \alpha T-3+4 e^{-\alpha T}+2 \alpha^{2} T^{2} e^{-\alpha T}-e^{-2 \alpha T}\right) \\
q_{12}= & q_{21}=\frac{1}{2 \alpha^{6}}\left(1-2 \alpha T+2 \alpha^{2} T^{2}-\alpha^{3} T^{3}+\frac{\alpha^{4} T^{4}}{4}\right. \\
& \left.+e^{-2 \alpha T}+2 \alpha T e^{-\alpha T}-2 e^{-\alpha T}-\alpha^{2} T^{2} e^{-\alpha T}\right) \\
q_{13}= & q_{31}=\frac{1}{2 \alpha^{5}}\left(2 \alpha T-\alpha^{2} T^{2}+\frac{\alpha^{3} T^{3}}{3}-3\right.
\end{aligned}
$$

\section{B. Measurement Equation and Its Linearization}

Let $\left(x_{k}, y_{k}, z_{k}\right)$ and $\left(r_{k}, \theta_{k}, \phi_{k}\right)$ be the respective Cartesian and spherical coordinates of the target at the $k$ th time sample. The basic radar measurements of the target position consists of its range $r_{k}$, azimuth angle $\theta_{k}$ and the elevation angle $\phi_{k}$. The following relations are used to transform the measurements from the polar (measurement) coordinates to the Cartesian (modeling) coordinates:

$$
\begin{aligned}
x_{k} & =r_{k} \cos \theta_{k} \cos \phi_{k} \\
y_{k} & =r_{k} \sin \theta_{k} \cos \phi_{k} \\
z_{k} & =r_{k} \sin \phi_{k}
\end{aligned}
$$

The equations in (4) can be inverted to yield

$$
\begin{aligned}
r_{k} & =\left(x_{k}^{2}+y_{k}^{2}+z_{k}^{2}\right)^{1 / 2} \\
\theta_{k} & =\tan ^{-1}\left(y_{k} / x_{k}\right) \\
\phi_{k} & =\tan ^{-1}\left(z_{k} / \rho_{k}\right)
\end{aligned}
$$

where $\rho_{k}=\left(x_{k}^{2}+y_{k}^{2}\right)^{1 / 2}$ is the projection of $r_{k}$ on the $x y$ plane (Fig. 1).

The next step is to linearize (14)-(16) in the Cartesian variables $x_{k}, y_{k}$, and $z_{k}$. From (5a) we obtain the partial derivatives: $\partial r_{k} / \partial x_{k}=x_{k} / r_{k}$, 
$\partial r_{k} / \partial y_{k}=y_{k} / r_{k}, \partial r_{k} / \partial z_{k}=z_{k} / r_{k}$. Expanding the right-hand side of (5a) in a Taylor series about the predicted position $\left(\hat{x}_{k \mid k-1}, \hat{y}_{k \mid k-1}, \hat{z}_{k \mid k-1}\right)$, and retaining terms up to the first derivative,

$$
\begin{aligned}
r_{k}= & \hat{r}_{k \mid k-1}+\left[\frac{\partial r_{k}}{\partial x_{k}}\right]_{x_{k}=\hat{x}_{k \mid k-1}}\left(x_{k}-\hat{x}_{k \mid k-1}\right) \\
& +\left[\frac{\partial r_{k}}{\partial y_{k}}\right]_{y_{k}=\hat{y}_{k \mid k-1}}\left(y_{k}-\hat{y}_{k \mid k-1}\right) \\
& +\left[\frac{\partial r_{k}}{\partial z_{k}}\right]_{z_{k}=\hat{z}_{k \mid k-1}}\left(z_{k}-\hat{z}_{k \mid k-1}\right) \\
= & \hat{r}_{k \mid k-1}+\frac{\hat{x}_{k \mid k-1}}{\hat{r}_{k \mid k-1}}\left(x_{k}-\hat{x}_{k \mid k-1}\right)+\frac{\hat{y}_{k \mid k-1}}{\hat{r}_{k \mid k-1}}\left(y_{k}-\hat{y}_{k \mid k-1}\right) \\
& +\frac{\hat{z}_{k \mid k-1}}{\hat{r}_{k \mid k-1}}\left(z_{k}-\hat{z}_{k \mid k-1}\right) \\
= & x_{k} \frac{\hat{x}_{k \mid k-1}}{\hat{r}_{k \mid k-1}}+y_{k} \frac{\hat{y}_{k \mid k-1}}{\hat{r}_{k \mid k-1}}+z_{k} \frac{\hat{z}_{k \mid k-1}}{\hat{r}_{k \mid k-1}}
\end{aligned}
$$

where $\hat{r}_{k \mid k-1}=\left(\hat{x}_{k \mid k-1}^{2}+\hat{y}_{k \mid k-1}^{2}+\hat{z}_{k \mid k-1}^{2}\right)^{1 / 2}$.

Similarly, from (5b) $\partial \theta_{k} / \partial x_{k}=-y_{k} / \rho_{k}^{2}$ and $\partial \theta_{k} / \partial y_{k}=x_{k} / \rho_{k}^{2}$, and hence

$$
\begin{aligned}
\theta_{k}= & \hat{\theta}_{k \mid k-1}+\left[\frac{\partial \theta_{k}}{\partial x_{k}}\right]_{x_{k}=\hat{x}_{k \mid k-1}}\left(x_{k}-\hat{x}_{k \mid k-1}\right) \\
& +\left[\frac{\partial \theta_{k}}{\partial y_{k}}\right]_{y_{k}=\hat{y}_{k \mid k-1}}\left(y_{k}-\hat{y}_{k \mid k-1}\right) \\
= & \tan ^{-1} \frac{\hat{y}_{k \mid k-1}}{\hat{x}_{k \mid k-1}}-x_{k} \frac{\hat{y}_{k \mid k-1}}{\hat{\rho}_{k \mid k-1}^{2}}+y_{k} \frac{\hat{x}_{k \mid k-1}}{\hat{\rho}_{k \mid k-1}^{2}}
\end{aligned}
$$

where $\hat{\rho}_{k \mid k-1}=\left(\hat{x}_{k \mid k-1}^{2}+\hat{y}_{k \mid k-1}^{2}\right)^{1 / 2}$. Finally, from (5c) $\partial \phi_{k} / \partial x_{k}=-x_{k} z_{k} / \rho_{k} r_{k}^{2}, \partial \phi_{k} / \partial y_{k}=-y_{k} z_{k} / \rho_{k} r_{k}^{2}$ and $\partial \phi_{k} / \partial z_{k}=\rho_{k} / r_{k}^{2}$, and hence

$$
\begin{aligned}
\phi_{k}= & \hat{\phi}_{k \mid k-1}+\left[\frac{\partial \phi_{k}}{\partial x_{k}}\right]_{x_{k}=\hat{x}_{k \mid k-1}}\left(x_{k}-\hat{x}_{k \mid k-1}\right) \\
& +\left[\frac{\partial \phi_{k}}{\partial y_{k}}\right]_{y_{k}=\hat{y}_{k \mid k-1}}\left(y_{k}-\hat{y}_{k \mid k-1}\right) \\
& +\left[\frac{\partial \phi_{k}}{\partial z_{k}}\right]_{z_{k}=\hat{z}_{k \mid k-1}}\left(z_{k}-\hat{z}_{k \mid k-1}\right) \\
= & \tan ^{-1} \frac{\hat{z}_{k \mid k-1}}{\hat{\rho}_{k \mid k-1}}-x_{k} \frac{\hat{z}_{k \mid k-1} \hat{x}_{k \mid k-1}}{\hat{\rho}_{k \mid k-1} \hat{r}_{k \mid k-1}^{2}} \\
& -y_{k} \frac{\hat{z}_{k \mid k-1} \hat{y}_{k \mid k-1}}{\hat{\rho}_{k \mid k-1} \hat{r}_{k \mid k-1}^{2}}+z_{k} \frac{\hat{\rho}_{k \mid k-1}}{\hat{r}_{k \mid k-1}^{2}}
\end{aligned}
$$

Equations (6), (7), and (8) represent linearized expressions for range, azimuth, and elevation, respectively, in the Cartesian coordinate system.
If we now define the vector $\mathbf{Z}_{J}$ of original measurements as

$$
\mathbf{Z}_{J: k+1}=\left[\begin{array}{c}
\text { measured range at }(k+1) \text { th instant } \\
\text { measured azimuth at }(k+1) \text { th instant } \\
\text { measured elevation at }(k+1) \text { th instant }
\end{array}\right]
$$

and the modified measurement vector $\mathbf{Z}_{J}^{*}$ as

$$
\begin{aligned}
\mathbf{Z}_{J: k+1}^{*}= & \mathbf{H}_{J: k+1} \mathbf{X}_{J: k+1} \\
& -\left[\begin{array}{lll}
0 & \tan ^{-1} \frac{\hat{y}_{k+1 \mid k}}{\hat{x}_{k+1 \mid k}} & \tan ^{-1} \frac{\hat{z}_{k+1 \mid k}}{\hat{\rho}_{k+1 \mid k}}
\end{array}\right]^{\mathrm{T}}
\end{aligned}
$$

then the measurement equation may be written as

$$
\mathbf{Z}_{J: k+1}^{*}=\mathbf{H}_{J: k+1} \mathbf{X}_{J: k+1}+\mathbf{V}_{J: k+1}
$$

where

$$
\begin{aligned}
& \mathbf{V}_{J: k+1}= \\
& {\left[\begin{array}{c}
\text { range: measurement noise at the }(k+1) \text { th instant } \\
\text { azimuth measurement noise at the }(k+1) \text { th instant } \\
\text { elevation measurement noise at the }(k+1) \text { th instant }
\end{array}\right]}
\end{aligned}
$$

$\mathbf{H}_{J: k+1}=\left[\begin{array}{cccccccccccc}h_{1} & 0 & 0 & 0 & h_{2} & 0 & 0 & 0 & h_{3} & 0 & 0 & 0 \\ h_{4} & 0 & 0 & 0 & h_{5} & 0 & 0 & 0 & 0 & 0 & 0 & 0 \\ h_{6} & 0 & 0 & 0 & h_{7} & 0 & 0 & 0 & h_{8} & 0 & 0 & 0\end{array}\right]$.

From equations (21), (22), (24), and (17)-(19), the non-zero elements of the matrix in (11b) are

$$
\begin{aligned}
& h_{1}=\hat{x}_{k+1 \mid k} / \hat{r}_{k+1 \mid k} \\
& h_{2}=\hat{y}_{k+1 \mid k} / \hat{r}_{k+1 \mid k} \\
& h_{3}=\hat{z}_{k+1 \mid k} / \hat{r}_{k+1 \mid k} \\
& h_{4}=-\hat{y}_{k+1 \mid k} / \hat{\rho}_{k+1 \mid k}^{2} \\
& h_{5}=\hat{x}_{k+1 \mid k} / \hat{\rho}_{k+1 \mid k}^{2} \\
& h_{6}=-\hat{x}_{k+1 \mid k} \hat{z}_{k+1 \mid k} /\left(\hat{r}_{k+1 \mid k}^{2} \hat{\rho}_{k+1 \mid k}\right) \\
& h_{7}=-\hat{y}_{k+1 \mid k} \hat{z}_{k+1 \mid k} /\left(\hat{r}_{k+1 \mid k}^{2} \hat{\rho}_{k+1 \mid k}\right) \\
& h_{8}=\hat{\rho}_{k+1 \mid k} / \hat{r}_{k+1 \mid k}^{2} .
\end{aligned}
$$

\section{ACCELERATION MODEL AND ACCELERATION FILTER}

Although the major focus in this work is on the derivation and study of a jerk model and a compatible tracking filter for 3-dimensional mixed coordinate tracking, we also study an acceleration model and the corresponding filter under identical tracking conditions for the purpose of comparison. Since the derivation of the acceleration model/filter equations 
proceeds in a manner parallel to the jerk filter, only the results are given here for the acceleration filter.

Using the subscript $A$ to denote the acceleration model/filter,

$$
\begin{aligned}
& \mathbf{X}_{A: k+1}=\mathbf{F}_{A} \mathbf{X}_{A: k}+\mathbf{W}_{A: k} \\
& \mathbf{Z}_{A: k+1}^{*}=\mathbf{H}_{A} \mathbf{X}_{A: k+1}+\mathbf{V}_{A: k+1}
\end{aligned}
$$

where

$$
\begin{aligned}
\mathbf{X}_{A} & =\left[\begin{array}{lllllllll}
x & \dot{x} & \ddot{x} & y & \dot{y} & \ddot{y} & z & \dot{z} & \ddot{z}
\end{array}\right]^{\mathrm{T}} \\
\mathbf{W}_{A} & =\left[\begin{array}{lllllllll}
u_{1 A} & u_{2 A} & u_{3 A} & u_{4 A} & u_{5 A} & u_{6 A} & u_{7 A} & u_{8 A} & u_{9 A}
\end{array}\right]^{\mathrm{T}} .
\end{aligned}
$$

The elements of (16) are the driving noise components for the state equation of the acceleration model. The modified measurement vector $\mathbf{Z}_{A}^{*}$ has a definition similar to (10).

The state transition matrix for the acceleration model is given by [5]

$$
\mathbf{F}_{A}=\left[\begin{array}{ccc}
\mathbf{T}^{\prime} & \mathbf{0} & \mathbf{0} \\
\mathbf{0} & \mathbf{T}^{\prime} & \mathbf{0} \\
\mathbf{0} & \mathbf{0} & \mathbf{T}^{\prime}
\end{array}\right]
$$

where 0 refers to a $3 \times 3$ null matrix, and

$$
\mathbf{T}^{\prime}=\left[\begin{array}{ccc}
1 & T & {\left[e^{-\alpha T}+\alpha T-1\right] / \alpha^{2}} \\
0 & 1 & {\left[1-e^{-\alpha T}\right] / \alpha} \\
0 & 0 & e^{-\alpha T}
\end{array}\right]
$$

and the noise covariance matrix by

$$
\mathbf{Q}_{A}=2 \alpha \sigma_{A}^{2}\left[\begin{array}{ccc}
\mathbf{Q}^{\prime} & \mathbf{0} & \mathbf{0} \\
\mathbf{0} & \mathbf{Q}^{\prime} & \mathbf{0} \\
\mathbf{0} & \mathbf{0} & \mathbf{Q}^{\prime}
\end{array}\right]
$$

where $\sigma_{A}^{2}$ is the variance, and $\alpha$ the correlation parameter, of the target acceleration. The elements of the $3 \times 3$ symmetric matrix $\mathbf{Q}^{\prime}$ are available in [2]. is

The measurement matrix for the acceleration filter

$$
\mathbf{H}_{A: k+1}=\left[\begin{array}{ccccccccc}
h_{1} & 0 & 0 & h_{2} & 0 & 0 & h_{3} & 0 & 0 \\
h_{4} & 0 & 0 & h_{5} & 0 & 0 & 0 & 0 & 0 \\
h_{6} & 0 & 0 & h_{7} & 0 & 0 & h_{8} & 0 & 0
\end{array}\right]
$$

with the elements $h_{1}$ though $h_{8}$ as given by (12).

\section{INITIALIZATION OF FILTERS}

The EKF in each case (jerk as well as acceleration models) is initialized by using a least-squares fit. A natural choice is to use a third-order polynomial for the jerk model and a second-order polynomial for the acceleration model, i.e.,

$$
\begin{aligned}
& \mathbf{r}(t)=\mathbf{b}_{0}+\mathbf{b}_{1} t+\mathbf{b}_{2} t^{2}+\mathbf{b}_{3} t^{3} \quad \text { (jerk model) } \\
& \mathbf{r}(t)=\mathbf{b}_{0}+\mathbf{b}_{1} t+\mathbf{b}_{2} t^{2} \quad \text { (acceleration model) }
\end{aligned}
$$

where $\mathbf{r}$ is the vector with components $x, y, z$, and the constants $\mathbf{b}_{0}, \mathbf{b}_{1}, \mathbf{b}_{2}$, and $\mathbf{b}_{3}$ are determined through the least-squares process. Following the derivation given in the Appendix, for the jerk model,

$$
\left[\begin{array}{c}
\mathbf{b}_{0}^{T} \\
\mathbf{b}_{1}^{T} \\
\mathbf{b}_{2}^{T} \\
\mathbf{b}_{3}^{T}
\end{array}\right]=
$$

$[\mathbf{L}]^{-1}\left[\begin{array}{ccc}\sum T(i-1) & \sum T(i-1) & \sum T(i-1) \\ \sum x_{m}(i) T(i-1) & \sum y_{m}(i) T(i-1) & \sum z_{m}(i) T(i-1) \\ \sum x_{m}(i)[T(i-1)]^{2} & \sum y_{m}(i)[T(i-1)]^{2} & \sum z_{m}(i)[T(i-1)]^{2} \\ \sum x_{m}(i)[T(i-1)]^{3} & \sum y_{m}(i)[T(i-1)]^{3} & \sum z_{m}(i)[T(i-1)]^{3}\end{array}\right]$

where

$[\mathbf{L}]=$

$$
\left[\begin{array}{cccc}
n & \sum T(i-1) & \sum[T(i-1)]^{2} & \sum[T(i-1)]^{3} \\
\sum T(i-1) & \sum[T(i-1)]^{2} & \sum[T(i-1)]^{3} & \sum[T(i-1)]^{4} \\
\sum[T(i-1)]^{2} & \sum[T(i-1)]^{3} & \sum[T(i-1)]^{4} & \sum[T(i-1)]^{5} \\
\sum[T(i-1)]^{3} & \sum[T(i-1)]^{4} & \sum[T(i-1)]^{5} & \sum[T(i-1)]^{6}
\end{array}\right]
$$

and, for the acceleration model,

$$
\left[\begin{array}{c}
\mathbf{b}_{0}^{T} \\
\mathbf{b}_{1}^{T} \\
\mathbf{b}_{2}^{T}
\end{array}\right]=
$$

$[\mathbf{L}]^{-1}\left[\begin{array}{ccc}\sum T(i-1) & \sum T(i-1) & \sum T(i-1) \\ \sum x_{m}(i) T(i-1) & \sum y_{m}(i) T(i-1) & \sum z_{m}(i) T(i-1) \\ \sum x_{m}(i)[T(i-1)]^{2} & \sum y_{m}(i)[T(i-1)]^{2} & \sum z_{m}(i)[T(i-1)]^{2}\end{array}\right]$

where

$$
[\mathbf{L}]=\left[\begin{array}{ccc}
n & \sum T(i-1) & \sum[T(i-1)]^{2} \\
\sum T(i-1) & \sum[T(i-1)]^{2} & \sum[T(i-1)]^{3} \\
\sum[T(i-1)]^{2} & \sum[T(i-1)]^{3} & \sum[T(i-1)]^{4}
\end{array}\right]
$$

Note that common symbols for the matrix $\mathbf{L}$ and the constants $\mathbf{b}_{0}, \mathbf{b}_{1}$, and $\mathbf{b}_{2}$ have been used in the two models only for the sake of notational uniformity; in general they will not have the same values in the two models. The variables $x_{m}, y_{m}, z_{m}$, in (23) and (24) refer to the measured values of $x, y, z$; in practice they are derived from the polar measurements $r_{m}, \theta_{m}, \phi_{m}$ by using (4).

\section{TRACKING PERFORMANCE OF MODELS}

Using the formulation developed in the earlier sections, the mixed-coordinate 3-dimensional tracking performance of the jerk and acceleration models/filters were evaluated through simulation. The parameters 
TABLE I

Simulation Parameters

\begin{tabular}{|c|c|c|c|}
\hline \multirow[b]{2}{*}{ Parameter } & \multicolumn{3}{|c|}{ Maneuver Level } \\
\hline & High & Medium & Low \\
\hline Angular velocity of the turn $\left(\mathrm{rad} \mathrm{s} \mathrm{s}^{-1}\right.$ ) & 0.1414 & 0.0971 & 0.0611 \\
\hline Peak velocity in $x$ and $y\left(\mathrm{~m} \mathrm{~s}^{-1}\right)$ & 495.0 & 339.7 & 214.0 \\
\hline Peak acceleration in $x$ and $y\left(\mathrm{~m} \mathrm{~s}^{-2}\right)$ & 70.0 & 33.0 & 13.0 \\
\hline Peak jerk in $x$ and $y\left(\mathrm{~m} \mathrm{~s}^{-3}\right)$ & 9.9 & 3.2 & 0.8 \\
\hline Standard deviation of range noise $\sigma_{r}(\mathrm{~m})$ & 10.0 & 10.0 & 10.0 \\
\hline Standard deviation of angle $(\mathrm{Az} / \mathrm{El})$ noise $\sigma_{\theta}, \sigma_{\phi}(\mathrm{mrad})$ & 5.0 & 5.0 & 5.0 \\
\hline$\alpha$ for acceleration model $\left(\mathrm{s}^{-1}\right)$ & 0.01 & 0.05 & 0.5 \\
\hline$\alpha$ for jerk model $\left(\mathrm{s}^{-1}\right)$ & 0.01 & 0.0001 & 0.0001 \\
\hline Process noise covariance for acceleration model $Q_{A}\left(\mathrm{~m}^{2} \mathrm{~s}^{-5}\right)$ & 100.0 & 20.0 & 2.0 \\
\hline Process noise covariance for jerk model $Q_{J}\left(\mathrm{~m}^{2} \mathrm{~s}^{-7}\right)$ & 2.0 & 0.2 & 0.03 \\
\hline
\end{tabular}

used for the simulation, and the comparative behavior of the two tracking filters, are outlined in this section.

\section{A. Trajectory Parameters}

The target is assumed to fly at constant speed along a circular trajectory of $3500 \mathrm{~m}$ radius with its center located at $x=y=5000 \mathrm{~m}$ relative to the tracker. The plane of the circle is at a constant height $z=1000 \mathrm{~m}$. The plan view of the trajectory is shown in Fig. 2. A circular trajectory has non-zero derivatives of all orders including jerk. For the trajectory chosen, the $x$ and $y$ position, velocity, acceleration, and jerk (as also the higher derivatives) are sinusoidal functions of time:

$$
\begin{aligned}
& x=r \cos \omega t \\
& y=r \sin \omega t \\
& \dot{x}=-\omega r \sin \omega t=v_{x} \\
& \text { and } \\
& \dot{y}=\omega r \cos \omega t=v_{y} \\
& \ddot{x}=-\omega^{2} r \cos \omega t=a_{x} \\
& \ddot{y}=-\omega^{2} r \sin \omega t=a_{y} \\
& \dddot{x}=\omega^{3} r \sin \omega t=j_{x} \\
& \dddot{y}=-\omega^{3} r \cos \omega t=j_{y}
\end{aligned}
$$

With such a trajectory, comparison of the steady state errors for different models is relatively straightforward as the pattern of maneuver is periodic and repetitive.

Monte Carlo simulation of tracker performance is carried out for three levels of target maneuver (high, medium, and low) induced by varying the flight speed of the target along the circular path of Fig. 2. The three levels correspond to peak lateral acceleration values of about $7 g, 3 g$, and $1 g$, respectively. For ' each maneuver level, both acceleration and jerk filters are run (for 3000 time samples at $0.1 \mathrm{~s}$ interval) for 50 sets of random noise samples, and their rms errors are compared. Optimum $\alpha$ and $Q$ values are chosen for best performance of each of the two filters as outlined in [5]. The parameters of simulation for the three maneuver levels are shown in Table I.

The parameters for the high maneuver trajectory are shown in Fig. 3 for the first 2000 time samples. As per (25) the $x$ and $y$ parameters are identical but for a phase shift, and this is apparent from the figure.

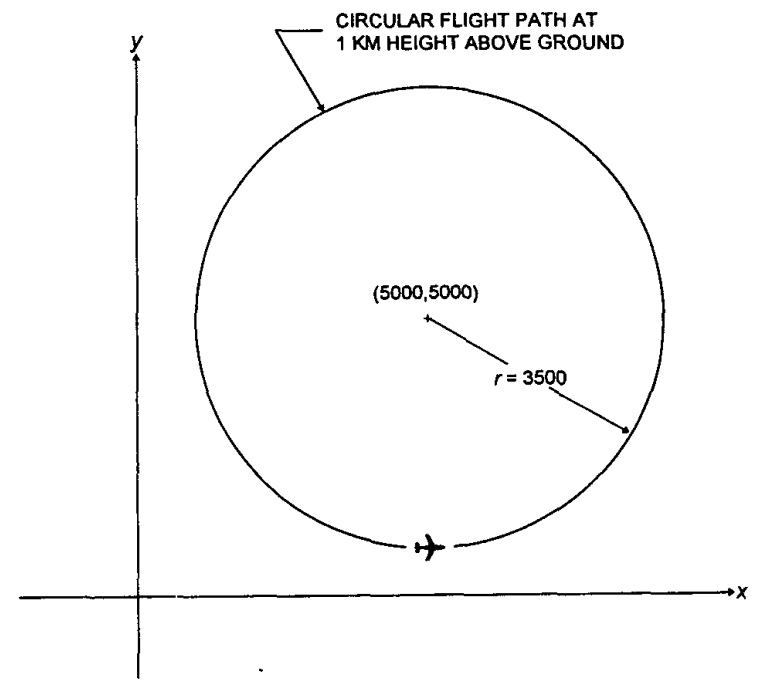

Fig. 2. Cartesian-coordinate trajectory parameters (in plan view, i.e., $x-y$ plane projection) of maneuvering target used for tracker performance evaluation.

The rms tracking errors of both acceleration and jerk models resulting from the Monte Carlo simulation are shown in Fig. 4, and the peak and average ${ }^{1}$ values of the steady-state errors over the tracking interval are shown in Table II. For all variables both peak and average estimation errors of the jerk filter (solid curves) are found to be lower than those of the acceleration filter (dotted curves) in the steady state. The advantage of the jerk filter is modest for the position variables, but is marked for the higher derivatives such as velocity and acceleration in $x$ and $y$. Along the $z$-axis the improvement due to the jerk model is modest for all derivatives, since it is a "passive" or no-maneuver axis. The cyclic fluctuations

\footnotetext{
I The errors being cyclic, the average value of finite data segments would be influenced by the data length. However, since the data here (we utilize 3000 time samples, which is longer than the time base shown in the figures) includes multiple cycles of variation, we use the mean value as an indicator for comparison of the two filters.
} 
TABLE II

Summary of Monte Carlo Simulation Results Shown in Fig. 4

\begin{tabular}{|c|c|c|c|c|}
\hline \multirow[t]{2}{*}{ Statistic $\rightarrow$} & \multicolumn{2}{|c|}{ Maximum* } & \multicolumn{2}{|c|}{ Mean* } \\
\hline & Jerk Model & Accn Model & Jerk Model & Accn Model \\
\hline$x$-position (m) & 20.7 & 25.1 & 11.1 & 12.0 \\
\hline$x$-velocity $\left(\mathrm{m} \mathrm{s}^{-1}\right)$ & 20.0 & 31.5 & 11.6 & 15.5 \\
\hline$x$-acceleration $\left(\mathrm{m} \mathrm{s}^{-2}\right)$ & 12.8 & 23.8 & 8.0 & 12.9 \\
\hline$x$-jerk $\left(\mathrm{m} \mathrm{s}^{-3}\right)$ & 5.8 & - & 3.6 & - \\
\hline$y$-position (m) & 22.5 & 22.6 & 10.6 & 11.4 \\
\hline$y$-velocity $\left(\mathrm{m} \mathrm{s}^{-1}\right)$ & 22.7 & 28.1 & 10.9 & 14.4 \\
\hline$y$-acceleration $\left(\mathrm{m} \mathrm{s}^{-2}\right)$ & 13.9 & 22.4 & 7.4 & 12.2 \\
\hline$y$-jerk $\left(\mathrm{m} \mathrm{s}^{-3}\right)$ & 5.8 & - & 3.4 & - \\
\hline$z$-position (m) & 21.9 & 23.3 & 13.2 & 14.3 \\
\hline Range $(\mathrm{m})$ & 6.1 & 6.9 & 4.5 & 5.0 \\
\hline Elevation (mrad) & 2.6 & 3.0 & 1.8 & 1.9 \\
\hline Azimuth (mrad) & 3.0 & 3.2 & 2.0 & 2.2 \\
\hline
\end{tabular}

Note: *The maximum and mean values have been computed without considering the initial transients.
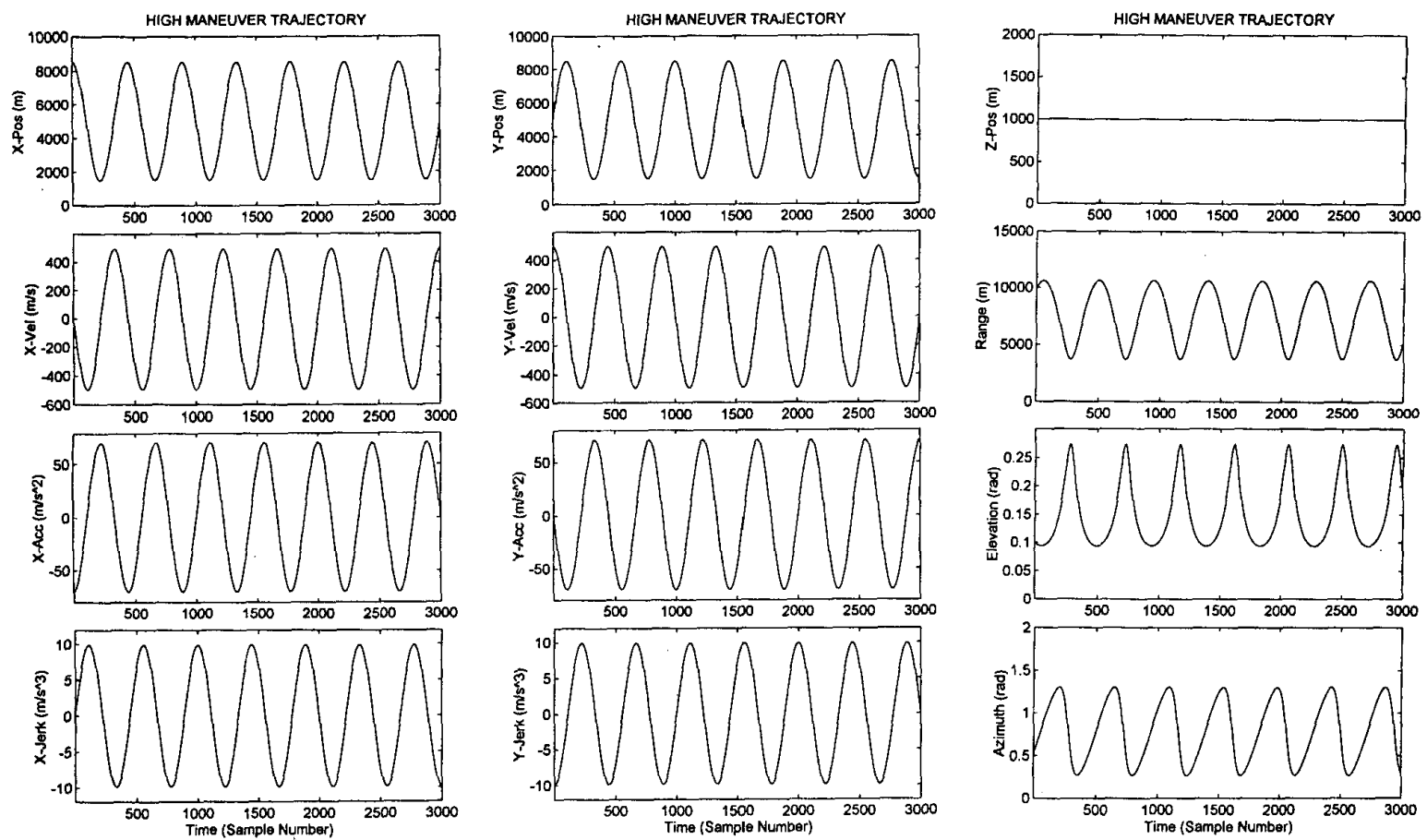

Fig. 3. Target position coordinates and their derivatives as functions of time for high-maneuvering situation.

in $z$-errors are due to coupling with $x$ and $y$ through the EKF. Note that the jerk error cannot be compared for the two filters, since the acceleration filter does not yield this estimate.

The initial values of the covariance matrix diagonal elements were chosen to be high for both filters, and the off-diagonal elements were assumed equal. The steady-state performance of the filter was found to be insensitive to these values. The initialization of the states was carried out as outlined in Section IV. The plots in Fig. 4 show that the initial transients are within manageable limits. The jerk filters exhibit relatively large transients, as expected due to the inclusion of a higher derivative.

It is possible to argue that the large advantage of the jerk filter in the above example is due to the presence of considerable jerk in the high maneuver trajectory. To compare the two models under less demanding conditions, we tested the two models also for medium and low maneuver (hence jerk) conditions with parameters as given in Table $\mathrm{I}$. These trajectories also show periodic parameter variations similar to Fig. 3. The Monte Carlo simulation results for these two maneuver levels are shown in Fig. 5 in 

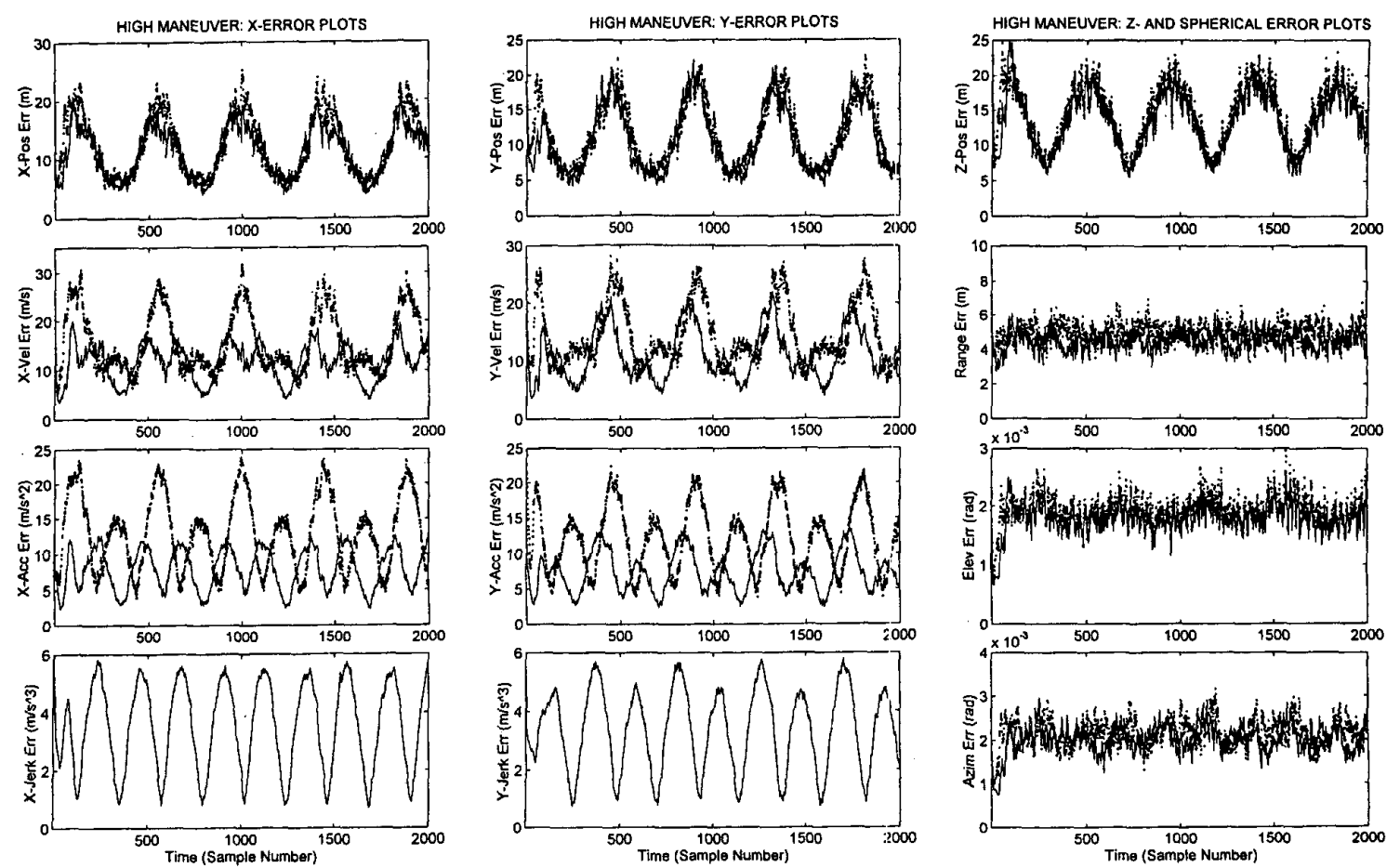

Fig. 4. Tracking errors in position coordinates and their derivatives for highly maneuvering target. $\cdots$ Acceleration filter, Jerk filter.

TABLE III

Summary of Monte Carlo Simulation Results Shown in Fig. 5

\begin{tabular}{|c|c|c|c|c|c|}
\hline \multirow{2}{*}{$\begin{array}{l}\text { Maneuver } \\
\text { Level }\end{array}$} & \multirow{2}{*}{ Error $\downarrow$} & \multicolumn{2}{|c|}{ Maximum* } & \multicolumn{2}{|c|}{ Mean* } \\
\hline & & Jerk Model & Accn Model & Jerk Model & Accn Model \\
\hline Low & $\begin{array}{l}x \text {-position }(\mathrm{m}) \\
x \text {-velocity }\left(\mathrm{m} \mathrm{s}^{-1}\right) \\
x \text {-acceleration }\left(\mathrm{m} \mathrm{s}^{-2}\right) \\
x \text {-jerk }\left(\mathrm{m} \mathrm{s}^{-3}\right)\end{array}$ & $\begin{array}{r}13.8 \\
6.0 \\
2.0 \\
0.4\end{array}$ & $\begin{array}{r}15.5 \\
9.7 \\
3.7 \\
-\end{array}$ & $\begin{array}{l}7.4 \\
3.8 \\
1.3 \\
0.3\end{array}$ & $\begin{array}{l}8.1 \\
5.1 \\
2.1 \\
-\end{array}$ \\
\hline Medium & $\begin{array}{l}x \text {-position }(\mathrm{m}) \\
x \text {-velocity }\left(\mathrm{m} \mathrm{s}^{-1}\right) \\
x \text {-acceleration }\left(\mathrm{m} \mathrm{s}^{-2}\right) \\
x \text {-jerk }\left(\mathrm{m} \mathrm{s}^{-3}\right)\end{array}$ & $\begin{array}{r}15.8 \\
11.5 \\
5.8 \\
1.8\end{array}$ & $\begin{array}{c}19.9 \\
18.2 \\
10.3 \\
-\end{array}$ & $\begin{array}{l}9.2 \\
6.9 \\
3.4 \\
1.1\end{array}$ & $\begin{array}{r}10.0 \\
9.3 \\
5.6 \\
-\end{array}$ \\
\hline
\end{tabular}

Note: ${ }^{*}$ The maximum and mean values have been computed without considering the initial transients.

an abridged form to minimize the number of plots, and a summary of results appears in Table III. The error plots clearly show that the jerk filter continues to be distinctly superior to the acceleration filter even for moderate to low maneuver and jerk levels.

\section{SENSITIVITY ANALYSIS}

The inclusion of higher derivatives has the potential of rendering models more sensitive to parameter variations relative to the optimum ones. To study this possibility, we simulated a model-filter mismatch situation. For space constraints, only the medium maneuver case ( $3 \mathrm{~g}$ circular maneuver) is reported here. The correlation parameter, which is 0.05 for the acceleration model in Table $\mathrm{I}$, was increased by a factor of 20 to 1.0 , and the $\alpha$ for the jerk model $(0.0001$ in Table I) was increased by an even larger factor of 100 , to a value of 0.01 . Representative results are shown in Fig. 6, and the peak and average errors are quoted in its caption. The top plot there compares the jerk and acceleration filters for the perturbed values of $\alpha$, and shows that the jerk model retains its marked superiority over the acceleration model. The bottom plot shows the jerk filter performance for the original (Table I) and perturbed $\alpha$ values to be nearly identical, testifying to the robustness of the filter even for very high departures of the model parameters. 

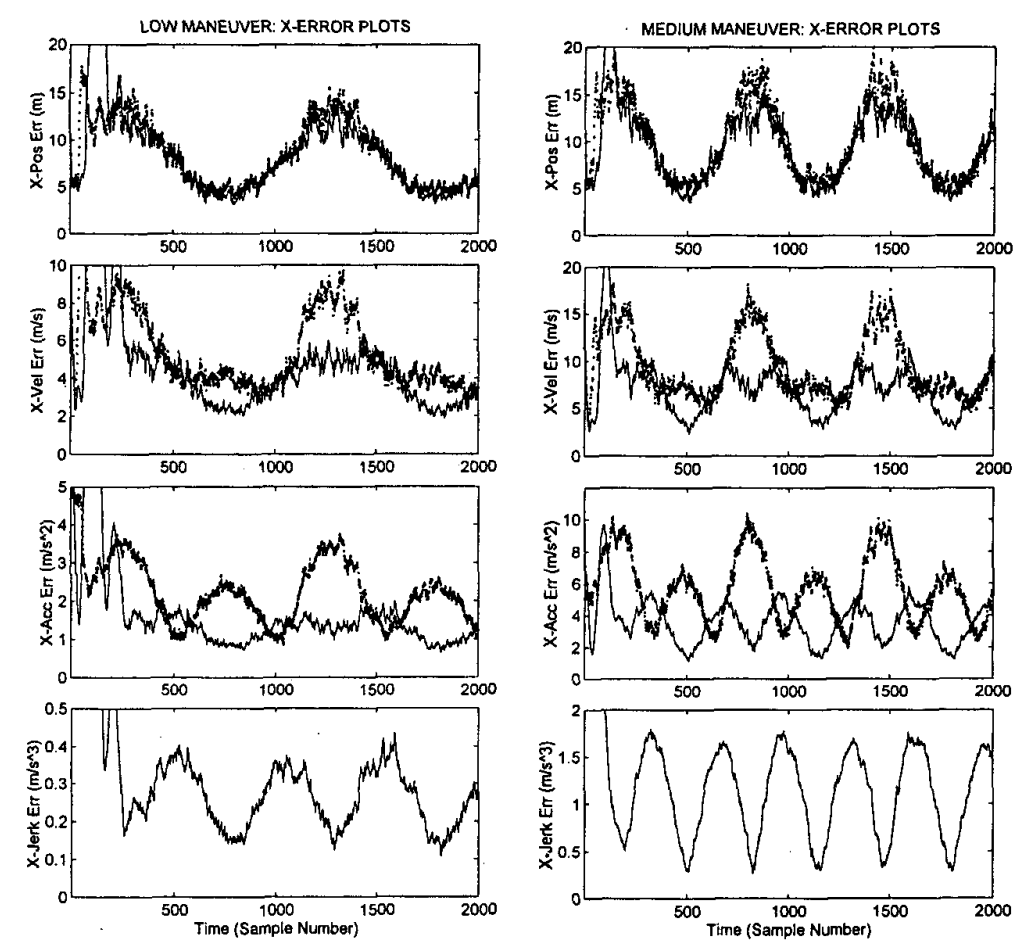

Fig. 5. Tracking errors in selected position coordinates and their derivatives for moderately maneuvering target (left column) and low-maneuvering target (right column). .... Acceleration filter, - Jerk filter.

\section{CONCLUDING REMARKS}

The main contribution of this work is to evolve the analytical framework for accurate tracking of generalized maneuvering targets in 3-dimensional space when the modeling of target motion and the measurements used for tracking purposes are in different types of coordinate systems. An enhanced target motion model is used which includes all terms up to its jerk. A realistic situation is considered where the target motion is described in a Cartesian coordinate system, while the measurements are performed in a polar coordinate system, as is normal with monostatic tracking radars. The resulting coordinate transformation introduces nonlinearities into the modeling process, which are handled through the EKF approach. The development here is complete, providing the equations as a handy reference to those interested in four-state tracking.

To show the advantage gained by the use of a jerk model/filter combination under mixed-coordinate tracking conditions, an acceleration model/filter is also derived under identical conditions. The 3-dimensional mixed-coordinate tracking performance of the jerk filter, and its parameter estimation accuracy vis-à-vis the acceleration filter, are demonstrated through Monte Carlo simulation using a circular target trajectory offset from the origin. The jerk filter is shown to perform distinctly better than the acceleration filter in terms of its tracking performance, especially when estimating the higher order motion derivatives of the
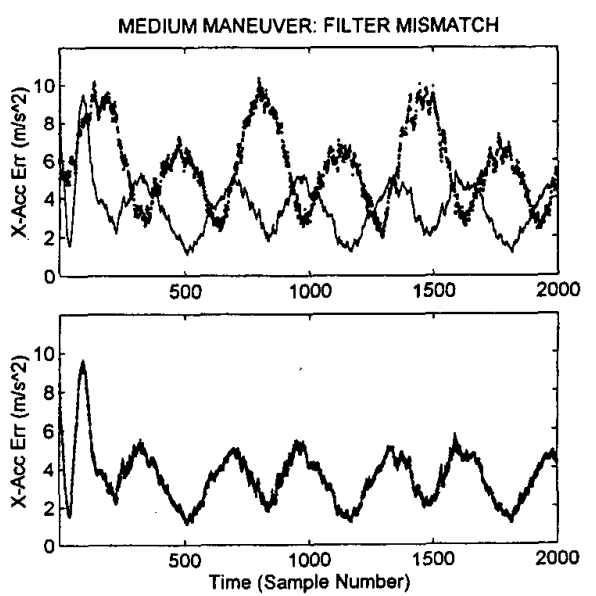

Fig. 6. Filter behavior for medium-maneuver target under model-filter mismatch conditions, showing $x$-acceleration plots: Top: mismatched jerk (-) and acceleration ( $(\ldots)$ filters; bottom: optimum (-) and mismatched $(\cdots)$ jerk filters. Note that solid and dotted curves nearly overlap in bottom plot, giving appearance of single curve. For jerk filter, $\alpha_{\text {opt }}=0.0001$,

$\alpha_{\text {mismatched }}=0.01$, and for acceleration filter, $\alpha_{\mathrm{opt}}=0.05$, $\alpha_{\text {mismatched }}=1.0$. Steady-state statistics of mismatched jerk filter (both top and bottom, units $\mathrm{m} \mathrm{s}^{-2}$ ) are: $\max =5.4$, mean $=3.4$; and for mismatched acceleration filter (top), $\max =10.3$, mean $=3.37$. Statistics of optimum acceleration filter (bottom) are as in Table III, i.e., $\max =5.8$, mean $=3.4$.

target. The simulation reveals that the advantages of the jerk filter can be very pronounced even when a small amount of jerk is present in the target maneuver. 


\section{APPENDIX}

We consider the acceleration model first.

Considering position, velocity, and acceleration terms, the equation of motion along the $x$ direction is

$$
x(t)=x(0)+t \dot{x}(0)+\left(\frac{t^{2}}{2}\right) \ddot{x}(0) .
$$

The constants $x(0), \dot{x}(0)$, and $\ddot{x}(0)$ in (26) correspond respectively to $b_{x 0}, b_{x 1}$, and $b_{x 2} / 2$ in a second-order polynomial equation in time

$$
x(t)=b_{x 0}+b_{x 1} t+b_{x 2} t^{2} .
$$

The task is to obtain the constants $b_{x 0}, b_{x 1}$, and $b_{x 2}$ which minimize the sum of the squared errors of the first $n$ measurements of $x$ with respect to (27). This is given by the equation (Kreyszig, 1993):

$$
\left[\begin{array}{ccc}
n & \sum t_{i} & \sum t_{i}^{2} \\
\sum t_{i} & \sum t_{i}^{2} & \sum t_{i}^{3} \\
\sum t_{i}^{2} & \sum t_{i}^{3} & \sum t_{i}^{4}
\end{array}\right]\left[\begin{array}{l}
b_{x 0} \\
b_{x 1} \\
b_{x 2}
\end{array}\right]=\left[\begin{array}{c}
\sum x_{i} \\
\sum x_{i} t_{i} \\
\sum x_{i} t_{i}^{2}
\end{array}\right]
$$

where $x_{i}$ is the $i$ th measurement of $x$ at the time instant $t_{i}$, and the summations are performed over the $n$ samples.

Equations similar to (28) can be written for the $y$ and $z$ axes. In normal practice, the first measurement sample $x_{1}$ is taken at the time instant $t_{i}=0$. Hence $t_{i}=T(i-1)$ where $T$ is the sampling interval. With this understanding, and noting that $\mathbf{b}_{k}=\left[\begin{array}{lll}b_{x k} & b_{y k} & b_{z k}\end{array}\right]^{\mathrm{T}}$ for $k=0,1,2,(24)$ is readily obtained.

For the jerk model, the equation of motion in the $x$ direction is

$$
x(t)=x(0)+t \dot{x}(0)+\left(\frac{t^{2}}{2}\right) \ddot{x}(0)+\left(\frac{t^{3}}{6}\right) \dddot{x}(0) .
$$

Writing the corresponding equations for the $y$ and $z$ directions, and following a procedure similar to the acceleration model above, the derivation of (23) is straightforward.

\section{ACKNOWLEDGMENT}

The authors gratefully acknowledge the numerous discussions with their colleague, Prof. M. R. Ananthasayanam, which lent clarity to some of the ideas.

PRAVAS R. MAHAPATRA

Department of Aerospace Engineering

Indian Institute of Science

Bangalore 560012, India

KISHORE MEHROTRA

Flat-1, 7 Brake Street

Upper Riccarton

Christchurch, New Zealand

\section{REFERENCES}

[1] Siouris, G. M., and Leros, A. P. (1988)

Minimum time intercept guidance for tactical missiles. Control Theory and Advanced Technology, 4 (June 1988) 251-263.

[2] Singer, R. A. (1970)

Estimating optimal tracking filter performance for manned maneuvering targets.

IEEE Transactions on Aerospace and Electronic Systems, AES-6 (July 1970), 473-483.

[3] Sudano, J. J. (1993)

The $\alpha-\beta-\Gamma$ tracking filter with a noisy jerk as the maneuver model.

IEEE Transactions on Aerospace and Electronic Systems, 29 (Aj)r. 1993), 578-580.

[4] Hoffman, S. A., and Blair, W. D. (1994)

Comments on $\alpha-\beta-\Gamma$ filter with a noisy jerk as the maneuver model.

IEEE Transactions on Aerospace and Electronic Systems, 30 (July 1994), 925-928.

[5] Mehrotra, K., and Mahapatra, P. R. (1997)

A jerk model for tracking highly maneuvering targets. IEEE Transactions on Aerospace and Electronic Systems, 33 (Oct. 1997).

[6] Kerr, T. H. (1991)

Strearnlining measurement iteration for extended Kalman filter.

IEEE Transactions on Aerospace and Electronic Systems, 27 (Mar. 1991), 408-421.

[7] Miller, K. S., and Leskiw, D. M. (1982)

Nonlinear estimation with radar observations.

IEEE Transactions on Aerospace and Electronic Systems, AES-18 (Mar. 1982), 192-200.

[8] Cortina, E., Otero, D., and D'Attelis, C. E. (1991) Maneuvering target tracking using extended Kalman filter. IEEE Transactions on Aerospace and Electronic Systems, 27 (Jan. 1991), 155-158.

[9] Kendrick, J. D., Maybeck, P. S., and Reid, J. G., (1981) Estimation of aircraft target motion using orientation measurements.

IEEE Transactions on Aerospace and Electronic Systems, AES-17 (Mar. 1981), 254-260.

[10] Lefas, C. C. (1984)

Using, roll angle measurements to track aircraft maneuvers.

IEEE Transactions on Aerospace and Electronic Systems, AES-20 (Nov. 1984), 672-681.

[11] Andrisani, D., Kuhl, F. P., and Gleason, D. (1986)

A nonlinear tracker using attitude measurements. IEEE Transactions on Aerospace and Electronic Systems, 22 (Sept. 1986), 533-539.

[12] Sworder, D. D., and Hutchins, R. G. (1990)

Maneuver estimation using measurements of orientation IEEE Transactions on Aerospace and Electronic Systems, 26 (July 1990), 625-638.

[13] Rekkas, C. M., Lefas, C. C., and Krikelis, N. J. (1991)

Three dimensional tracking using on-board measurements.

IEEE Transactions on Aerospace and Electronic Systems, 27 (July 1991), 617-624.

[14] Kreyszig (1993)

Advanced Engineering Mathematics (7th ed.).

New York: Wiley, 1993, p. 1002, eq. (5) 